\title{
CpG Island Methylator Phenotype is Associated With Response to Adjuvant Irinotecan-Based Therapy for Stage 3 Colon Cancer
}

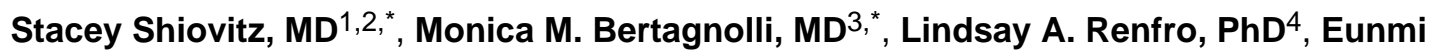 \\ Nam, MD, PhD ${ }^{5}$, Nathan R. Foster, MS $^{4}$, Slavomir Dzieciatkowski, BS ${ }^{2}$, Yanxin Luo, MD, \\ $\mathrm{PhD}^{2,6}$, Victoria Valinluck Lao, MD, PhD ${ }^{2,7}$, Raymond J. Monnat Jr., MD ${ }^{8}$, Mary J. Emond, \\ PhD $^{9}$, Nancy Maizels, PhD ${ }^{10}$, Donna Niedzwiecki, PhD ${ }^{11}$, Richard M. Goldberg, MD ${ }^{12}$, \\ Leonard B. Saltz, MD ${ }^{13}$, Alan Venook, MD ${ }^{14}$, Robert S. Warren, MD ${ }^{14}$, William M. Grady, \\ $\mathbf{M D}^{1,2}$, and the Alliance for Clinical Trials in Oncology \\ ${ }^{1}$ Department of Medicine, University of Washington (UW) \\ ${ }^{2}$ Clinical Research Division, Fred Hutchinson Cancer Research Center \\ ${ }^{3}$ Dana-Farber Cancer Institute and Brigham and Women's Hospital \\ ${ }^{4}$ Alliance Statistics and Data Center, Mayo Clinic \\ ${ }^{5}$ Division of Hematology/Oncology, Ewha Womans University School of Medicine, South Korea \\ ${ }^{6}$ Department of Colorectal Surgery, The Sixth Affiliated Hospital, Sun Yat-Sen University, P.R. \\ China \\ ${ }^{7}$ Department of Surgery, UW Medical School \\ ${ }^{8}$ Departments of Pathology and Genome Science, UW Medical School \\ ${ }^{9}$ Department of Biostatistics, UW
}

\footnotetext{
(C) 2014 The American Gastroenterological Association. Published by Elsevier Inc. All rights reserved. (206) 667-1107; wgrady@fhcrc.org, fax: (206) 667-2917.

Co-first authors

Publisher's Disclaimer: This is a PDF file of an unedited manuscript that has been accepted for publication. As a service to our the resulting proof before it is published in its final citable form. Please note that during the production process errors may be discovered which could affect the content, and all legal disclaimers that apply to the journal pertain.

Author contributions:

- $\quad$ Study concept and design: $\mathrm{MMB}, \mathrm{WMG}$

- $\quad$ Acquisition of data: EN, SD, YL, VVL

- Analysis and interpretation of data: SS, MMB, LAR, NRF, MJE, WMG

- Drafting of the manuscript: all authors

- Critical revision of the manuscript for important intellectual content: all authors

- $\quad$ Obtained funding: MMB, WMG, RJM, LBS, RSW, NRF, SS, DN, LAR, AV, VVL, RMG

- Administrative, technical, or material support: MMB, RMG, AV, LBS, RSW

- $\quad$ Study supervision: MMB, WMG
}

Correspondence: William M. Grady; Fred Hutchinson Cancer Research Center, 1100 Fairview Ave N. D4-100, Seattle, WA 98109; customers we are providing this early version of the manuscript. The manuscript will undergo copyediting, typesetting, and review of

Conflicts of interest: None

Author names in bold designate shared co-first authorship. 
${ }^{10}$ Departments of Immunology and Biochemistry, UW

${ }^{11}$ Alliance Statistics and Data Center, Duke University Medical Center

${ }^{12}$ The Ohio State University

${ }^{13}$ Memorial Sloan-Kettering Cancer Center

${ }^{14}$ University of California-San Francisco and the Helen Diller Family Comprehensive Cancer Center

\section{Abstract}

BACKGROUND \& AIMS-The CpG island methylator phenotype (CIMP), defined by a high frequency of aberrantly methylated genes, is a characteristic of a subclass of colon tumors with distinct clinical and molecular features. Cohort studies have produced conflicting results on responses of CIMP-positive tumors to chemotherapy. We assessed the association between tumor CIMP status and survival of patients receiving adjuvant fluorouracil and leucovorin alone or with irinotecan (IFL)

METHODS-We analyzed data from patients with stage 3 colon adenocarcinoma randomly assigned to groups given fluorouracil and leucovorin or IFL following surgery, from April 1999 through April 2001. The primary endpoint of the trial was overall survival and the secondary endpoint was disease-free survival. DNA isolated from available tumor samples $(\mathrm{n}=615)$ was used to determine CIMP status based on methylation patterns at the CACNAIG, IGF2, NEUROG1, RUNX3, and SOCS1 loci. The effects of CIMP on survival were modeled using Kaplan-Meier and Cox proportional hazards; interactions with treatment and $B R A F, K R A S$, and mismatch repair (MMR) status were also investigated.

RESULTS-Of the tumor samples characterized for CIMP status, 145 were CIMP positive (23\%). Patients with CIMP-positive tumors had shorter overall survival times than patients with CIMP-negative tumors (hazard ratio $[\mathrm{HR}]=1.36$; 95\% confidence interval $[\mathrm{CI}], 1.01-1.84$ ). Treatment with IFL showed a trend toward increased overall survival for patients with CIMPpositive tumors, compared to treatment with fluorouracil and leucovorin (HR=0.62; 95\% CI, $0.37-$ $1.05 ; P=.07$ ), but not for patients with CIMP-negative tumors (HR=1.38; 95\% CI, 1.00-1.89; $P=$. 049). In a 3-way interaction analysis, patients with CIMP-positive, MMR-intact tumors benefited most from the addition of irinotecan to fluorouracil and leucovorin therapy (for the interaction, $P=.01)$. CIMP was more strongly associated with response to IFL than MMR status. Results for disease-free survival times were comparable among all analyses.

CONCLUSION-Patients with stage 3, CIMP-positive, MMR-intact colon tumors have longer survival times when irinotecan is added to combination therapy with fluorouracil and leucovorin.

\section{Keywords}

CALGB (Alliance) 89803; CRC; epigenetic factors; chemotherapy 


\section{INTRODUCTION}

Colorectal cancer (CRC) is a complex disease, demonstrating substantial molecular heterogeneity as reflected by the wide array of gene mutations and epigenetic alterations in the average CRC genome. ${ }^{1}$ Some of these alterations appear to have prognostic or predictive value, such as mismatch repair deficiency (MMR-D), also known as microsatellite instability (MSI) and BRAF mutations. ${ }^{1-3}$ Fluorouracil/leucovorin (FU/LV) and oxaliplatin combination chemotherapy is the standard of care following resection of stage III colon cancer, resulting in an improvement in overall survival. ${ }^{4}$ While irinotecan does provide benefit in metastatic CRC, ${ }^{5}$ it has not been shown to be effective in unselected patients for adjuvant treatment. ${ }^{6}$ In this era of precision medicine, it is important to determine whether there is a subgroup of patients that would benefit from adjuvant FU/LV with irinotecan.

In the Cancer and Leukemia Group B/Alliance 89803 (C89803) trial, patients with stage III adenocarcinoma of the colon were randomized to adjuvant weekly FU/LV alone versus in combination with irinotecan (IFL). No aggregate overall survival benefit of IFL treatment was observed in this trial. ${ }^{6}$ In a subset of 506 patients there was worse overall survival for patients with tumors with mutant $B R A F$ (hazard ratio (HR) vs. wild-type 1.66, 95\% confidence interval (CI): 1.05-2.63, $P=.015$ ). The worst prognostic group had $B R A F$-mutant, mismatch repair intact (MMR-I) tumors, while the most favorable group had wild-type $B R A F$, MMR-D tumors. ${ }^{3}$

Additional analysis of C89803 showed that mismatch repair-deficiency (MMR-D) associates with improved five-year overall and disease-free survival ( $76 \%$ vs. $59 \%, P=.03$ ), whereas loss of heterozygosity $(\mathrm{LOH})$ at $18 \mathrm{q}$ was not predictive of an unfavorable outcome. ${ }^{7,8}$ However, the effect of MMR-D was not confirmed in a separate randomized trial with a similar study cohort and treatment (PETACC-3) ${ }^{9}$ Additionally, in CRC cell lines, correction of MMR deficiency did not reverse the differential sensitivity to irinotecan, suggesting that tumor MMR-D alone does not explain these results. ${ }^{10}$ Thus, it has been postulated that the increased sensitivity could be mediated by a mismatch repair-associated mechanism. ${ }^{11}$

A molecular alteration commonly observed in MMR-D CRC is the CpG Island Methylator Phenotype (CIMP), characterized by a high frequency of genome-wide aberrant DNA methylation of $\mathrm{CpG}$ islands. ${ }^{12}$ Observed in about $20 \%$ of CRC, when present, CIMP is believed to promote carcinogenesis by the silencing of tumor suppressor genes secondary to methylation-mediated transcriptional repression. MMR-D due to hypermethylation is now thought of as a subset of CIMP with the most common cause being aberrant hypermethylation of $M L H 1 .{ }^{12-15}$ Occurring more often in women and older individuals, CIMP-positive tumors typically are proximal, higher grade, and have MMR-D, wild-type TP53, and mutant BRAF. ${ }^{13-18}$

Like MMR-D, ${ }^{7,19}$ CIMP status has also been evaluated as a predictive marker for chemotherapy responsiveness. There are conflicting data regarding whether CIMP-positive tumors receive benefit from adjuvant FU. ${ }^{18,20-23}$ Possible explanations for this inconsistency include the use of small case-control studies, differences in the loci used to define CIMP, and different hypermethylation assays used. To address these issues, in an 
exploratory analysis, we studied the association of CIMP with response to FU/LV versus IFL using a validated ${ }^{13,16}$ assay panel for CIMP and samples drawn from a large randomized phase III trial.

\section{MATERIALS AND METHODS}

\section{Characteristics of Study Population}

Following curative surgical resection of histologically confirmed stage III colon adenocarcinoma, patients were enrolled between 4/1999 and 4/2001 and then randomized (fixed block) to receive either FU/LV or IFL under C89803. ${ }^{6}$ Patients with known diagnoses of Lynch syndrome or Familial Adenomatous Polyposis (FAP) were excluded from this trial. The primary end point was overall survival, measured from clinical trial entry until death from any cause. Disease-free survival, defined as the time from study entry until the earlier of documented progression of disease or death from any cause, was a secondary endpoint. Additional secondary aims addressed the relationship between tumor-associated risk factors and treatment outcome. This protocol was approved by the Institutional Review Board of each center and the studies in this manuscript received IRB approval from the FHCRC (IR 1989). All patients consented to participation in this study and for molecular analysis. The CALGB Statistical Center maintains the clinical and laboratory database with follow-up completed on 11/9/09.

The current analysis is limited to 615 patients for whom archived formalin-fixed paraffinembedded (FFPE) tumor tissue was available for DNA extraction. Protocols for molecular testing of TP53, BRAF, and KRAS and MMR have been described previously. 3, 7, 24, 25

\section{Treatment}

As previously published, ${ }^{6}$ FU/LV treatment consisted of weekly LV $500 \mathrm{mg} / \mathrm{m}^{2}$ intravenously (IV) over 2 hours, with bolus FU $500 \mathrm{mg} / \mathrm{m}^{2}$ IV 1 hour after initiation of LV for a total of four cycles or 32 weeks of therapy. The IFL group received irinotecan $125 \mathrm{mg} / \mathrm{m}^{2}$ IV over 90 minutes, LV $20 \mathrm{mg} / \mathrm{m}^{2}$ IV bolus, then FU $500 \mathrm{mg} / \mathrm{m}^{2}$ IV bolus for five cycles or 30 weeks. In the primary endpoint analysis, the 5-year overall survival probability was 0.71 (95\% CI: $0.67-0.75 ; 201$ events in 629 patients) in the FU/LV arm and 0.68 (95\% CI: 0.64-0.72; 221 events in 635 patients) in the IFL arm with a median follow-up of 4.8 years. ${ }^{6}$

\section{DNA extraction from tumor}

Tumor molecular analyses were carried out blinded to patient and outcome data. DNA was extracted from FFPE tissue using Bio-Rad's InstaGene Matrix. To enrich for tumor epithelium and confirm the histological diagnosis, hematoxylin \& eosin-stained slides from all cases were reviewed and marked. The corresponding area in adjacent sections were identified and microdissected using sterile razor blades to achieve $>70 \%$ tumor, and then subjected to DNA extraction. The assays were performed in a non-CLIA approved research laboratory at the Fred Hutchinson Cancer Research Center (PI: Grady). 


\section{Sodium Bisulfite Conversion and Sample Preparation}

Sodium bisulfite conversion of approximately 1.0 $\mu \mathrm{g}$ genomic DNA was performed using

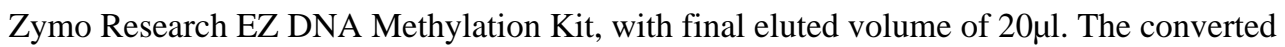
DNA was diluted 1:10 for MethyLight analysis, whereas methylated and unmethylated controls (CpGenome Universal Methylated/Unmethylated DNA from Millipore) were diluted 1:80. A total of 5 $\mu \mathrm{l}$ of diluted DNA was used per PCR reaction. Additionally, serial dilutions of the methylated control DNA (Millipore) were included on each PCR assay plate for standard curve generation.

\section{MethyLight Analysis of Five CIMP-Specific Markers}

Following sodium bisulfite treatment, genomic DNA was analyzed by MethyLight using a Bio-Rad CFX96 Real-Time System. These results were scored as PMR (Percent of Methylated Reference) values. The primer and probe sequences for the MethyLight reactions are as follows: CACNA1G Forward:TTTTTTCGTTTCGCGTTTAGGT, Reverse:CTCGAAACGACTTCGCCG, Probe:6FAMAAATAACGCCGAATCCGACAACCGA-MGBNFQ. IGF2 Forward:GAGCGGTTTCGGTGTCGTTA, Reverse:CCAACTCGATTTAAACCGACG, Probe:6FAM-CCCTCTACCGTCGCGAACCCGA-MGBNFQ. NEUROG1 Forward:CGTGTAGCGTTCGGGTATTTGTA, Reverse:CGATAATTACGAACACACTCCGAAT, Probe: 6FAMCGATAACGACCTCCCGCGAACATAAA-MGBNFQ. RUNX3 Forward:CGTTCGATGGTGGACGTGT, Reverse:GACGAACAACGTCTTATTACAACGC, Probe: 6FAMCGCACGAACTCGCCTACGTAATCCG-MGBNFQ. SOCS1 Forward:GCGTCGAGTTCGTGGGTATTT, Reverse:CCGAAACCATCTTCACGCTAA, Probe:6FAM-ACAATTCCGCTAACGACTATCGCGCA-MGBNFQ. AluC4 Forward:GGTTAGGTATAGTGGTTTATATTTGTAATTTTAGTA, Reverse:ATTAACTAAACTAATCTTAAACTCCTAACCTCA, Probe:6FAMCCTACCTTAACCTCCC-MGBNFQ.

PCR amplification was performed using Bio-Rad Hard-Shell Thin-Wall 96-Well Skirted

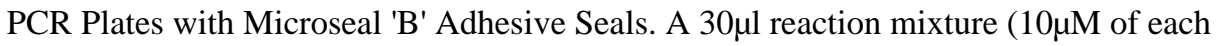
primer, $3 \mu \mathrm{M}$ probe, $6 \mathrm{mM}$ dNTP mix, $3 \mu \mathrm{l}$ of HotStarTaq 10x Buffer (Qiagen), 105mM $\mathrm{MgCl}_{2}, 1.5 \mathrm{U}$ HotStarTaq DNA Polymerase (Qiagen), and $5 \mu \mathrm{l}$ bisulfite-converted DNA) was cycled under the following conditions: $95^{\circ} \mathrm{C}$ for $15 \mathrm{~min}$., followed by 50 cycles of $95^{\circ} \mathrm{C}$ for $15 \mathrm{sec}$. and $60^{\circ} \mathrm{C}$ for $1 \mathrm{~min}$.

Methylation of $\mathrm{CpG}$ islands was evaluated for a validated group of five sites in the following genes: CACNA1G, IGF2, NEUROG1, RUNX3, and SOCS1. ${ }^{13,16}$ The PMR was set at $4 \%$ per literature standards, ${ }^{26}$ with "CIMP-positive" defined as $\_4 \%$ methylation in at least three of five tested $\mathrm{CpG}$ islands.

\section{Statistical Methods}

Prognostic relationships between tumor CIMP status and outcomes were modeled using Cox proportional hazards regression, while predictive effects of CIMP with treatment were 
examined through Cox model interaction testing. Cox models were further used to adjust for clinicopathologic factors: age (continuous), sex, tumor stage, tumor grade, performance status (PS) (Eastern Cooperative Oncology Group (ECOG) 0 vs. 1-2), number of positive lymph nodes (continuous), and location of primary tumor. Also considered were possible CIMP interactions with and adjustment for other markers ( $K R A S, B R A F$, and $\mathrm{MMR}^{8}$ status). Interactions associated with p-values $<0.05$ were further examined for clinical relevance using Kaplan-Meier methods and subgroup analyses. Log-rank tests were used for survival comparisons between categories defined by CIMP status or treatment, while Likelihood ratio p-values were reported for multivariable Cox regression models. The Kaplan-Meier method was used to estimate the overall and disease-free survival curves and associated five-year overall and disease-free survival probabilities. For these analyses, follow-up was limited to eight years, where patients not experiencing events by eight years were rightcensored. All statistical analyses were performed by Alliance statisticians.

\section{RESULTS}

Of the 1264 patients in C89803, 615 had tumor DNA available for CIMP analysis. Of these, $419(67 \%)$ had results for all 5 CIMP markers and $463(74 \%)$ had results for at least 3 CIMP markers (Individual marker results for each case can be found in Figure S1.). The CIMP analyzed group was well matched to the overall cohort except for being slightly older (median age 63 vs. 59 years, $P=.011$; full details in online supplementary Table S1). There was no significant difference between the two groups regarding randomized treatment arm, gender, PS, tumor site (distal vs. proximal), tumor T-stage, histologic grade, number of positive lymph nodes, MMR status, or prevalence of mutations in BRAF, KRAS, or TP53. In addition, disease-free survival did not differ significantly between subjects with or without available CIMP data (HR=1.00, 95\%CI: 0.85-1.19, $P=.978)$.

\section{CIMP Relationship with Clinical Factors and Biomarkers}

Patients with CIMP-positive tumors (Table 1) were similar to the published clinical phenotype, ${ }^{13-18}$ with older age of onset and tumors that were more proximal, higher grade, MMR-D, $B R A F$-mutant, and $K R A S$-wild-type as compared to patients with CIMP-negative tumors (see Figure 1). No difference was seen between CIMP-positive and CIMP-negative groups by gender or TP53 mutation status. The patients with CIMP-positive tumors had a slightly worse PS (ECOG 0: 72.7\% CIMP-positive vs. 76.5\% CIMP-negative patients, $P=$. 03).

\section{Relationship between CIMP and patient outcomes}

As shown in Figure 2A, patients with CIMP-positive tumors had worse overall survival $(\mathrm{HR}=1.36,95 \% \mathrm{CI}: 1.01-1.84 ; P=.044)$. In a univariate analysis, five-year overall survival was $63.0 \%$ (95\% CI: 55.0-71.0\%) for CIMP-positive patients and 73.3\% (95\%CI: 69.2-77.4\%) for CIMP-negative patients. However, the CIMP effect became non-significant after adjustment for treatment and patient variables including those (age, PS) distributed unevenly by CIMP status $(P=.31)$. CIMP status was not associated with disease-free survival (Figure 2B, $P=.0 .28$ ), either unadjusted or when adjusted for treatment and clinical variables. 


\section{Relationship between CIMP and response to treatment}

A two-way interaction model between treatment arm and CIMP status indicated that survival after adjuvant chemotherapy differed by CIMP status (interaction $P=.01$ ), and that this interaction remained significant after adjustment for clinical variables (adjusted interaction, $P=.002)$. Within the FU/LV arm (n=316), patients with CIMP-positive (vs. negative) tumors experienced worse overall survival (HR=2.06, 95\%CI: 1.36-3.11, $P=$. 0005). In contrast, in the IFL arm ( $\mathrm{n}=299)$, patients with CIMP-positive tumors had overall improved outcomes and no significant difference was observed compared to CIMP-negative tumors (HR=0.90, 95\%CI: 0.58-1.41, $P=.65$ ) (Figure 3A). Looking at the same interaction another way, patients with CIMP-positive tumors demonstrated a trend toward improved overall survival when treated with IFL versus FU/LV (69\% vs. 56\%, respectively; 95\%CI: 0.37-1.05, $P=.07)$. In contrast, among patients with CIMP-negative tumors, worse overall survival was observed for IFL versus FU/LV (68\% vs. 78\%; HR=1.38, 95\%CI: 1.00-1.89, $P=.049$ ). Disease-free survival results were similar (Figure 3B, adjusted interaction $P=.007$ ).

\section{Relationship between CIMP and MMR, KRAS, BRAF}

Separate overall survival analyses of MMR (using IHC where available and genotyping otherwise $^{8}$; $\left.\mathrm{n}=910\right)$ and CIMP ( $\left.\mathrm{n}=615\right)$ in C89803 revealed that CIMP was a much stronger predictor for response to IFL therapy than MMR status (interaction $P=.01$ vs. $P=.52$, respectively). When both CIMP and MMR were included in a two-variable model, CIMP adjusted for MMR showed a stronger effect on overall survival $(P=.006)$ than MMR adjusted for CIMP $(P=.06)$, indicating that CIMP is the more important prognostic factor. Given the known association between MMR-D and CIMP in colon cancer, we next determined whether there was an interaction between MMR-D and CIMP that associated with outcome. This interaction was significant for both overall $(P=.007)$ and disease-free survival $(P=.005)$, and remained significant after adjustment for treatment and clinical variables (overall survival $P=.005$; disease-free survival $P=.008$ ). Specifically, patients with CIMP-positive tumors that were also MMR-D had improved overall survival compared to the patients with CIMP-positive, MMR-I tumors (HR=0.42, 95\%CI: 0.23-0.77, $P=.004$ ) (Figure 4A). Among patients with CIMP-positive tumors, the estimated five-year survival rate in the MMR-D group was $74.6 \%$ versus $54.7 \%$ in patients with MMR-I tumors. However, there was no significant difference in overall survival between MMR groups within patients with CIMP-negative tumors (HR=1.38, 95\%CI: 0.74-2.55; $P=.31$ ). Diseasefree survival results were similar (Figure 4B), and both overall and disease-free survival relationships remained significant when adjusted for treatment and clinicopathologic variables.

We also assessed possible relationships between CIMP and other previously published biomarkers from C89803. In a two-variable Cox model for overall survival containing both CIMP and $K R A S$, CIMP remained a marginally significant predictor of overall survival $(P=$. 04) after adjustment by $K R A S$, while $K R A S$ was not significant in the same model after adjustment for CIMP $(P=.77)$. CIMP and $K R A S$ were jointly non-significant in a two variable model for disease-free survival. In the two-variable Cox model with CIMP and $B R A F$, these were jointly non-significant for both overall and disease-free survival. We separately conducted two-way models of CIMP interaction with $B R A F$ and $K R A S$, but found 
no significant interactions in either unadjusted or adjusted models for overall and diseasefree survival (data not shown).

\section{Three-way interaction of CIMP, MMR, and Treatment}

Given the strong two-way interactions reported between CIMP and treatment as well as between CIMP and MMR, we investigated a possible three-way interaction between CIMP status, MMR status, and treatment arm (Figure 5). This interaction was significant for both overall $(P=.01)$ and disease-free survival $(P=.02)$ and remained significant when adjusted for clinical and pathologic variables (overall survival $P=.01$; disease-free survival $P=.01$; full details in supplemental online Table S2). For patients receiving FU/LV (Figure 5A), improved overall survival was seen in two groups of patients: those whose tumors were CIMP-negative, MMR-I and those whose tumors were CIMP-positive, MMR-D. (Of note, this latter group contained only 21 patients with 4 events, which limits the robustness of this result.) By comparison, CIMP-negative, MMR-D ( $\mathrm{n}=17,8$ events) and CIMP-positive, MMR-I groups treated with FU/LV demonstrated worse overall survival. Specifically, among MMR-I patients treated with FU/LV, CIMP-positive patients showed significantly worse overall survival than CIMP-negative patients (five-year overall survival: $46 \%$ vs. 79\%, respectively; HR=3.11, 95\%CI: 1.98-4.90; $<<0.0001$ ), while the CIMP effect on overall survival among MMR-D, FU/LV-treated patients trended in the opposite direction (five-year overall survival: $80 \%$ vs. $57 \%$, respectively; $\mathrm{HR}=0.34,95 \% \mathrm{CI}: 0.10-1.12 ; P=.06$ ). In contrast, among patients treated with IFL (Figure 5B), there was no significant difference between subgroups defined by CIMP and MMR status. From these results, it is evident that the group with the greatest irinotecan benefit was poor prognosis patients with CIMPpositive, MMR-I tumors. Within this group (albeit a smaller $n=80$ ), treatment with IFL versus FU/LV was associated with a five-year overall survival of $66 \%$ vs. $46 \%$, respectively, and a HR of 0.53 (95\%CI: $0.28-1.01 ; P=.048$ ).

Describing the same interaction another way (Figure 5C, 5D), we found that in patients with CIMP-negative tumors, worse overall survival was observed in the FU/LV treatment arm among patients with MMR-D vs. MMR-I tumors (five-year overall survival: 57\% vs. 79\%, respectively; HR=2.22, 95\% CI: 1.06-4.65; $P=.03$ ). In contrast, in FU/LV-treated patients with CIMP-positive tumors, improved overall survival was observed in patients with MMRD vs. MMR-I tumors (five-year overall survival: $80 \%$ vs. $46 \%$; HR $=0.23,95 \% \mathrm{CI}$ : $0.08-0.67 ; P=.003)$. No significant survival difference by MMR status was observed among IFL-treated patients in either the CIMP-negative or CIMP-positive groups.

The overall survival results have been reported in detail given that the goal of treating stage III patients is with curative intent. Disease-free survival results were similar to overall survival. Specifically, between MMR-I, FU/LV-treated patients, tumors that were CIMPpositive (vs. negative) were associated with worse disease-free survival (HR=2.36, 95\% CI: 1.53-3.63, $\mathrm{p}<0.0001$ ), while the CIMP trend among MMR-D, FU/LV-treated patients was in the opposite direction (HR=0.31, 95\%CI: 0.11-0.89, $P=.02$ ). Among FU/LV-treated patients with CIMP-positive tumors, MMR-D (vs. MMR-I) was associated with improved diseasefree survival (HR=0.28, 95\%CI: 0.11-0.73, $P=.006)$. However, among CIMP-negative patients treated with FU/LV, a trend in the opposite direction was apparent (MMR-D vs. 
MMR-I; HR: 2.14, 95\%CI: 1.14-4.03, $P=.02)$. A significant benefit from IFL was not exhibited in subgroups defined by CIMP and MMR.

\section{DISCUSSION}

In this exploratory analysis of stage III colon cancer patients in a prospective, randomized trial of FU/LV versus IFL, patients with CIMP-negative tumors had better unadjusted survival than those with CIMP-positive tumors. Patients with CIMP-positive tumors treated with IFL experienced a trend toward improved survival compared to patients with CIMPpositive tumors who received FU/LV alone. Patients with CIMP-negative tumors, however, exhibited significant harm from the addition of irinotecan. Among patients treated with FU/LV alone, those with CIMP-positive (vs. negative) tumors demonstrated significantly improved overall survival. As overall survival is the ultimate goal of treatment, this was the highlighted outcome. We note, however, that disease-free survival closely mirrored the overall survival results, consistent with CIMP being a predictive marker for IFL response and lessening the likelihood that subsequent therapies negatively impacted the predictive utility of CIMP.

While patients with MMR-D (vs. MMR-I) tumors had consistently better outcomes, MMR status was a weaker predictor for IFL response than CIMP, with statistical significance for CIMP compared to non-significance for MMR. We speculate that the difference in the predictive strength of MMR reported in the prior study ${ }^{7}$ and the current study is because the present analysis utilizes more patients under an updated MMR definition, ${ }^{8}$ and considers events over the entirety of the follow-up period rather than with a statistical dichotomization (event or no-event) at the five-year time point only. Indeed, when all available follow-up information is used and the endpoint is not dichotomized, the $P$-value for the two-way interaction between marker and treatment arm is 0.01 for CIMP and 0.56 for MMR, with the latter indicating no predictive effect of MMR. Results are similar for predictive analyses for disease-free survival and when adjusted by clinical variables. Collectively, this CIMP analysis, combined with the previously published MMR results, ${ }^{7,8}$ serves to increase our understanding of which subset of patients might benefit from IFL adjuvant therapy as compared to FU/LV. Patients with CIMP-positive tumors had better survival if the tumors were also MMR-D; the worst outcome was seen for patients whose tumors were both CIMPpositive and MMR-I. No overall survival difference was observed by MMR status among CIMP-negative patients.

The irinotecan treatment benefit in patients with CIMP-positive tumors was largely driven by tumors that were additionally classified as MMR-I. In this poor prognosis group of patients with CIMP-positive, MMR-I tumors, treatment with IFL was associated with improved overall survival over 8 years of follow-up compared to the same group treated with FU/LV (borderline statistical significance, $P=.051$ ). Given the small number of patients in this exploratory subgroup, validation of these results is necessary in a larger, independent cohort. The even smaller subgroup of patients with CIMP-negative, MMR-D tumors also appeared to benefit from IFL, but the small numbers limited any firm conclusions at this point. No significant associations or interactions between CIMP and KRAS or BRAF 
mutations were observed, suggesting that the effectiveness of FU/LV and IFL are not influenced by KRAS/BRAF mutation status.

The etiology of the CIMP phenotype remains unknown, limiting our understanding of the molecular mechanisms underpinning chemotherapy responsiveness in these CRCs. We observed that patients with CIMP-positive tumors had worse overall survival compared to patients with CIMP-negative tumors both overall and within the FU/LV treatment group. While not universally demonstrated, our results are consistent with prior studies showing no benefit or an adverse response to FU-based adjuvant chemotherapy in patients with CIMPpositive tumors. ${ }^{18,20-22,27,28}$ Thus, we propose that CIMP status has significant potential for a greater role in the clinical setting. In addition to these results, there is also emerging data that demethylating agents may be of benefit for solid tumors as well as CIMP-positive CRCs. ${ }^{29,30}$

Our results suggest that patients with stage III CIMP-positive (specifically, CIMP-positive, MMR-I) colon cancer may have greater benefit from adjuvant IFL compared to FU/LV. We acknowledge that these results must be considered exploratory in nature given that evaluation of CIMP responsiveness was not a pre-determined endpoint of this study. To our knowledge, however, prior to this study, there are minimal published data available to evaluate irinotecan response by CIMP status, with only one small trial reporting results for patients with metastatic disease. ${ }^{31}$

A clear mechanistic understanding to explain the better outcome of CIMP-positive patients is currently lacking. Abnormalities in global DNA methylation may be responsible for altering chemotherapy responsiveness of CIMP-positive CRC. In support of this, CRCs with high (vs. low) levels of LINE-1 methylation had better prognosis, but were not affected by FU. ${ }^{32}$ There may be additional genes differentially expressed in CIMP-positive vs. negative $\mathrm{CRC}$ that are responsible for the disparate chemotherapy responses. Mechanisms that warrant investigation relate to the activity status of DNA repair enzymes that are involved in single-strand break repair (e.g. RECQ helicases) and base excision repair (e.g. OGG1, MYH). ${ }^{33,} 34$

In summary, we found that patients with stage III CIMP-positive colon cancer had a trend toward improved overall survival when treated with adjuvant IFL compared to FU/LV. In addition, patients with CIMP-positive colon cancer who were treated with FU/LV had decreased overall survival compared to the other subgroups (i.e., CIMP-positive treated with IFL or CIMP-negative regardless of treatment arm). We observed an interaction between MMR status and CIMP status in relation to overall survival; CIMP-positive, MMR-D cases exhibited the best overall survival over 8 years of follow-up and CIMP-positive, MMR-I cases exhibited the worst overall survival. Our results suggest that irinotecan may provide benefit when added to FU/LV for the adjuvant therapy of stage III CIMP-positive, MMR-I colon cancer. Our results do not address whether irinotecan with FU is more effective than the standard combination of oxaliplatin and FU or whether CIMP-positive, MMR-I metastatic patients would have greater benefit from first-line irinotecan. However, they suggest that for patients with CIMP-positive colon cancers who may be unable to tolerate oxaliplatin, irinotecan plus FU/LV may be a reasonable treatment strategy. 


\section{Supplementary Material}

Refer to Web version on PubMed Central for supplementary material.

\section{Acknowledgments}

We thank Dan Rohrer in the CALGB Pathology Coordinating Office for processing the samples and providing the tissue sections for the cases.

Grant support: Research reported in this manuscript was supported by National Institutes of Health (NIH) National Cancer Institute (NCI) Program award P01CA77852 (RJM); NIH awards 5T32-CA009515-28/29 (SS), RO1CA115513, P30CA15704, UO1CA152756, U54CA143862, and P01CA077852 (WMG); Burroughs Wellcome Fund Translational Research Award for Clinician Scientist (WMG); ACS fellowship PF-11-086-01-TBG, NIH 2T32DK007742-16, ASCRS GSRRIG, and NIH NCI F32CA1591555-01 (VVL); CA32291 (MMB); CA77658 (RMG); CA77651 (LBS); CA60138 (AV,RSW). CALGB 89803 (Alliance) was supported partly by grants from the NCI (CA31946) to the Alliance for Clinical Trials in Oncology (MMB, Chair) and to the Alliance Statistics and Data Center (Daniel J. Sargent, Ph.D., CA33601) (LAR,NRF,DN). The content is solely the responsibility of the authors and does not necessarily represent the official views of the NIH.

\section{Abbreviations}

CI Confidence interval

CIMP $\quad$ CpG Island Methylator Phenotype

CRC Colorectal cancer

ECOG Eastern Cooperative Oncology Group

FFPE Formalin-fixed paraffin-embedded

HR

Hazard ratio

IFL Irinotecan, fluorouracil, and leucovorin

FU Fluorouracil

LOH Loss of heterozygosity

LV Leucovorin

MMR Mismatch repair

MMR-D Mismatch repair-deficient

MMR-I Mismatch repair-intact

MSI Microsatellite instability

MSI-H Microsatellite instability-high

MSI-L Microsatellite instability-low

MSS Microsatellite stable

PMR Percent methylated reference

PS Performance status 


\section{REFERENCES}

1. Walther A, Johnstone E, Swanton C, et al. Genetic prognostic and predictive markers in colorectal cancer. Nat Rev Cancer. 2009; 9:489-99. [PubMed: 19536109]

2. Popat S, Hubner R, Houlston RS. Systematic review of microsatellite instability and colorectal cancer prognosis. J Clin Oncol. 2005; 23:609-18. [PubMed: 15659508]

3. Ogino S, Shima K, Meyerhardt JA, et al. Predictive and prognostic roles of BRAF mutation in stage III colon cancer: results from intergroup trial CALGB 89803. Clin Cancer Res. 2012; 18:890-900. [PubMed: 22147942]

4. Andre T, Boni C, Mounedji-Boudiaf L, et al. Oxaliplatin, fluorouracil, and leucovorin as adjuvant treatment for colon cancer. N Engl J Med. 2004; 350:2343-51. [PubMed: 15175436]

5. Saltz LB, Cox JV, Blanke C, et al. Irinotecan plus fluorouracil and leucovorin for metastatic colorectal cancer. Irinotecan Study Group. N Engl J Med. 2000; 343:905-14. [PubMed: 11006366]

6. Saltz LB, Niedzwiecki D, Hollis D, et al. Irinotecan fluorouracil plus leucovorin is not superior to fluorouracil plus leucovorin alone as adjuvant treatment for stage III colon cancer: results of CALGB 89803. J Clin Oncol. 2007; 25:3456-61. [PubMed: 17687149]

7. Bertagnolli MM, Niedzwiecki D, Compton CC, et al. Microsatellite instability predicts improved response to adjuvant therapy with irinotecan, fluorouracil, and leucovorin in stage III colon cancer: Cancer and Leukemia Group B Protocol 89803. J Clin Oncol. 2009; 27:1814-21. [PubMed: 19273709]

8. Bertagnolli MM, Redston M, Compton CC, et al. Microsatellite instability and loss of heterozygosity at chromosomal location 18q: prospective evaluation of biomarkers for stages II and III colon cancer--a study of CALGB 9581 and 89803. J Clin Oncol. 2011; 29:3153-62. [PubMed: 21747089]

9. Roth AD, Tejpar S, Delorenzi M, et al. Prognostic role of KRAS and BRAF in stage II and III resected colon cancer: results of the translational study on the PETACC-3, EORTC 40993, SAKK 60-00 trial. J Clin Oncol. 2010; 28:466-74. [PubMed: 20008640]

10. Mohindra A, Hays LE, Phillips EN, et al. Defects in homologous recombination repair in mismatch-repair-deficient tumour cell lines. Hum Mol Genet. 2002; 11:2189-200. [PubMed: 12189171]

11. Sharma S, Doherty KM, Brosh RM Jr. Mechanisms of RecQ helicases in pathways of DNA metabolism and maintenance of genomic stability. Biochem J. 2006; 398:319-37. [PubMed: 16925525]

12. Grady WM, Carethers JM. Genomic and epigenetic instability in colorectal cancer pathogenesis. Gastroenterology. 2008; 135:1079-99. [PubMed: 18773902]

13. Ogino S, Kawasaki T, Kirkner GJ, et al. Evaluation of markers for $\mathrm{CpG}$ island methylator phenotype (CIMP) in colorectal cancer by a large population-based sample. J Mol Diagn. 2007; 9:305-14. [PubMed: 17591929]

14. Toyota M, Ahuja N, Ohe-Toyota M, et al. CpG island methylator phenotype in colorectal cancer. Proc Natl Acad Sci U S A. 1999; 96:8681-6. [PubMed: 10411935]

15. Hawkins N, Norrie M, Cheong K, et al. CpG island methylation in sporadic colorectal cancers and its relationship to microsatellite instability. Gastroenterology. 2002; 122:1376-87. [PubMed: 11984524]

16. Weisenberger DJ, Siegmund KD, Campan M, et al. CpG island methylator phenotype underlies sporadic microsatellite instability and is tightly associated with BRAF mutation in colorectal cancer. Nat Genet. 2006; 38:787-93. [PubMed: 16804544]

17. van Rijnsoever M, Grieu F, Elsaleh H, et al. Characterisation of colorectal cancers showing hypermethylation at multiple CpG islands. Gut. 2002; 51:797-802. [PubMed: 12427779]

18. Min BH, Bae JM, Lee EJ, et al. The CpG island methylator phenotype may confer a survival benefit in patients with stage II or III colorectal carcinomas receiving fluoropyrimidine-based adjuvant chemotherapy. BMC Cancer. 2011; 11:344. [PubMed: 21827707]

19. Pritchard CC, Grady WM. Colorectal cancer molecular biology moves into clinical practice. Gut. 2011; 60:116-29. [PubMed: 20921207] 
20. Van Rijnsoever M, Elsaleh H, Joseph D, et al. CpG island methylator phenotype is an independent predictor of survival benefit from 5-fluorouracil in stage III colorectal cancer. Clin Cancer Res. 2003; 9:2898-903. [PubMed: 12912934]

21. Jover R, Nguyen TP, Perez-Carbonell L, et al. 5-Fluorouracil adjuvant chemotherapy does not increase survival in patients with $\mathrm{CpG}$ island methylator phenotype colorectal cancer. Gastroenterology. 2011; 140:1174-81. [PubMed: 21185836]

22. Shen L, Catalano PJ, Benson AB 3rd, et al. Association between DNA methylation and shortened survival in patients with advanced colorectal cancer treated with 5-fluorouracil based chemotherapy. Clin Cancer Res. 2007; 13:6093-8. [PubMed: 17947473]

23. Ward RL, Cheong K, Ku SL, et al. Adverse prognostic effect of methylation in colorectal cancer is reversed by microsatellite instability. J Clin Oncol. 2003; 21:3729-36. [PubMed: 14551292]

24. Ogino S, Meyerhardt JA, Irahara N, et al. KRAS mutation in stage III colon cancer and clinical outcome following intergroup trial CALGB 89803. Clin Cancer Res. 2009; 15:7322-9. [PubMed: 19934290]

25. Warren RS, Atreya CE, Niedzwiecki D, et al. Association of TP53 Mutational Status and Gender with Survival After Adjuvant Treatment for Stage III Colon Cancer: Results of CALGB 89803. Clin Cancer Res. 2013

26. Ogino S, Kawasaki T, Brahmandam M, et al. Precision and performance characteristics of bisulfite conversion and real-time PCR (MethyLight) for quantitative DNA methylation analysis. J Mol Diagn. 2006; 8:209-17. [PubMed: 16645207]

27. Iacopetta B, Kawakami K, Watanabe T. Predicting clinical outcome of 5-fluorouracil-based chemotherapy for colon cancer patients: is the $\mathrm{CpG}$ island methylator phenotype the 5fluorouracil-responsive subgroup? Int J Clin Oncol. 2008; 13:498-503. [PubMed: 19093176]

28. Anderson GL, Limacher M, Assaf AR, et al. Effects of conjugated equine estrogen in postmenopausal women with hysterectomy: the Women's Health Initiative randomized controlled trial. JAMA. 2004; 291:1701-12. [PubMed: 15082697]

29. Zouridis H, Deng N, Ivanova T, et al. Methylation subtypes and large-scale epigenetic alterations in gastric cancer. Sci Transl Med. 2012; 4:156ra140.

30. Garrido-Laguna I, McGregor K, Wade M, et al. Testing targeted demethylation to overcome resistance to epidermal growth factor receptor (EGFR) blocking agents in wild-type (wt) KRAS metastatic colorectal cancer patients (mCRC). J Clin Oncol. 2012; 30(suppl) abstr 3057.

31. Ogino S, Meyerhardt JA, Kawasaki T, et al. CpG island methylation, response to combination chemotherapy, and patient survival in advanced microsatellite stable colorectal carcinoma. Virchows Arch. 2007; 450:529-37. [PubMed: 17372756]

32. Kawakami K, Matsunoki A, Kaneko M, et al. Long interspersed nuclear element-1 hypomethylation is a potential biomarker for the prediction of response to oral fluoropyrimidines in microsatellite stable and $\mathrm{CpG}$ island methylator phenotype-negative colorectal cancer. Cancer Sci. 2011; 102:166-74. [PubMed: 21087350]

33. Bohr VA. Rising from the RecQ-age: the role of human RecQ helicases in genome maintenance. Trends Biochem Sci. 2008; 33:609-20. [PubMed: 18926708]

34. Kennedy RD, D'Andrea AD. DNA repair pathways in clinical practice: lessons from pediatric cancer susceptibility syndromes. J Clin Oncol. 2006; 24:3799-808. [PubMed: 16896009] 
CIMP-positive ( $\mathrm{n}=145)$

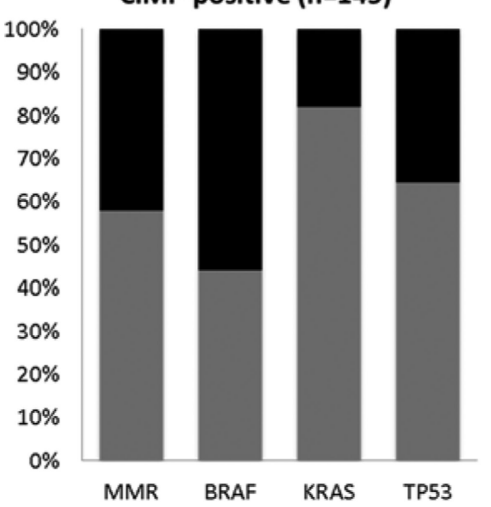

CIMP-negative $(n=470)$

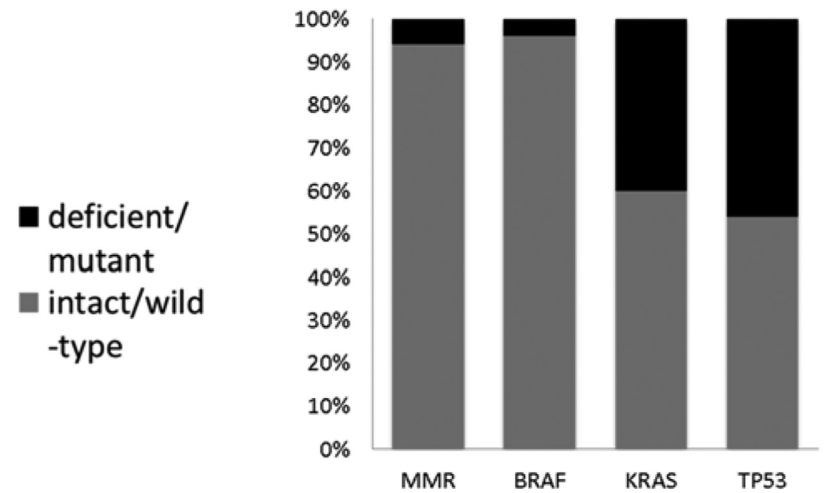

Figure 1.

CIMP-positive tumors were more often MMR-deficient, $B R A F$-mutated, $K R A S$-wild-type compared to CIMP-negative tumors. The frequencies of TP53 mutations were similar in the CIMP-positive and CIMP-negative groups. 
A: Overall Survival in CIMP-positive and CIMP-negative CRC

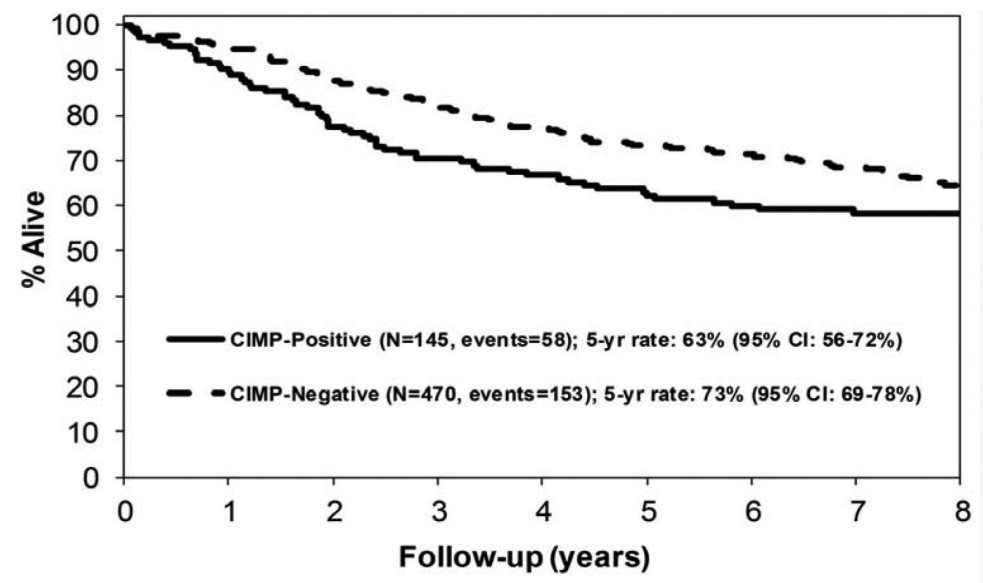

\section{B: Disease-Free Survival in CIMP-positive and CIMP-negative CRC}

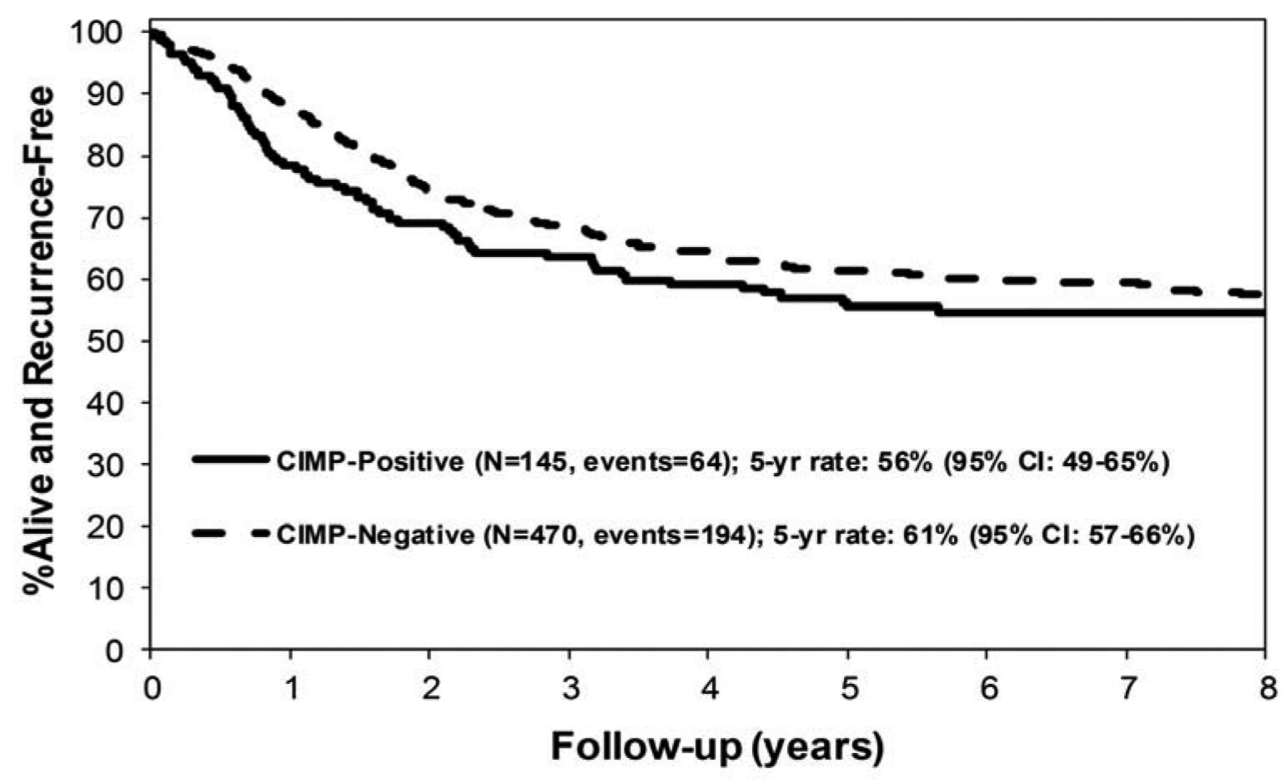

Figure 2.

Patients with CIMP-positive tumors had significantly worse overall survival compared to patients with CIMP-negative tumors. CIMP status alone was not associated with diseasefree survival. 


\section{A: Interaction of CIMP status and treatment for Overall Survival}

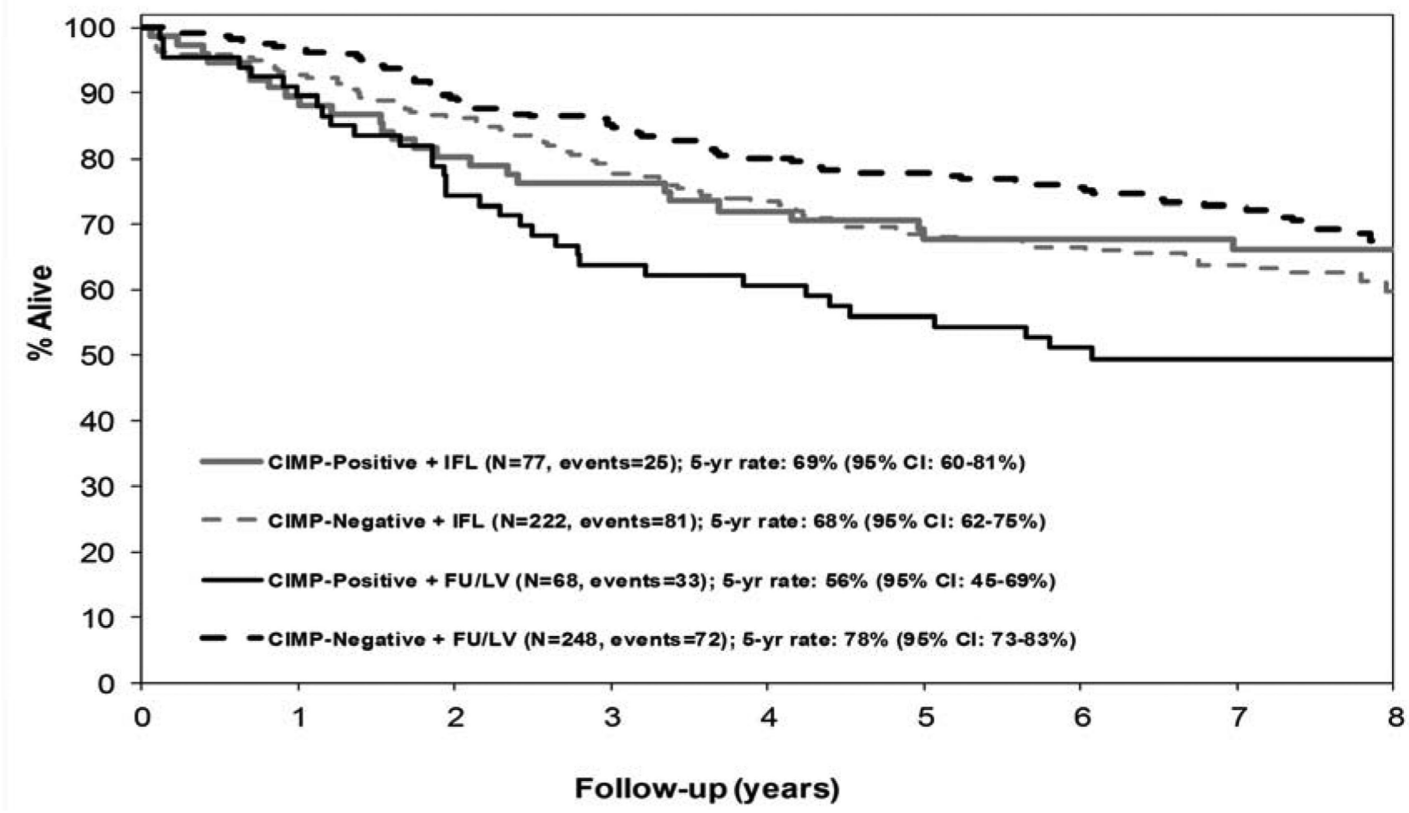

\section{B: Interaction of CIMP status and treatment for Disease-Free Survival}

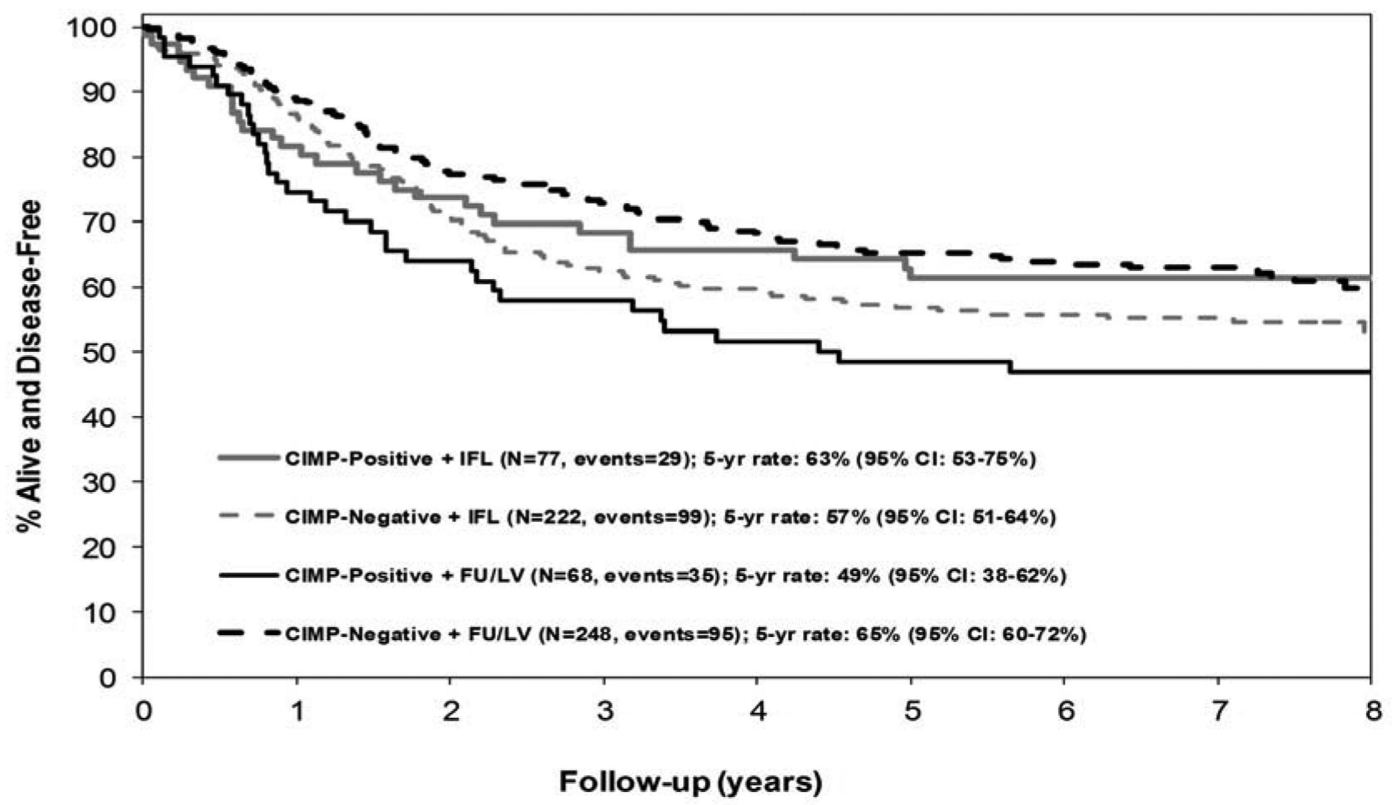

Figure 3. 
In the two-way interaction model between treatment arm and CIMP status, of patients treated with FU/LV, CIMP-positivity was associated with worse overall survival; whereas in patients treated with IFL, there was no overall survival difference between CIMP-positive and CIMP-negative tumors. Disease-free survival results were similar. 


\section{A: Interaction of CIMP status and MMR status for Overall Survival}

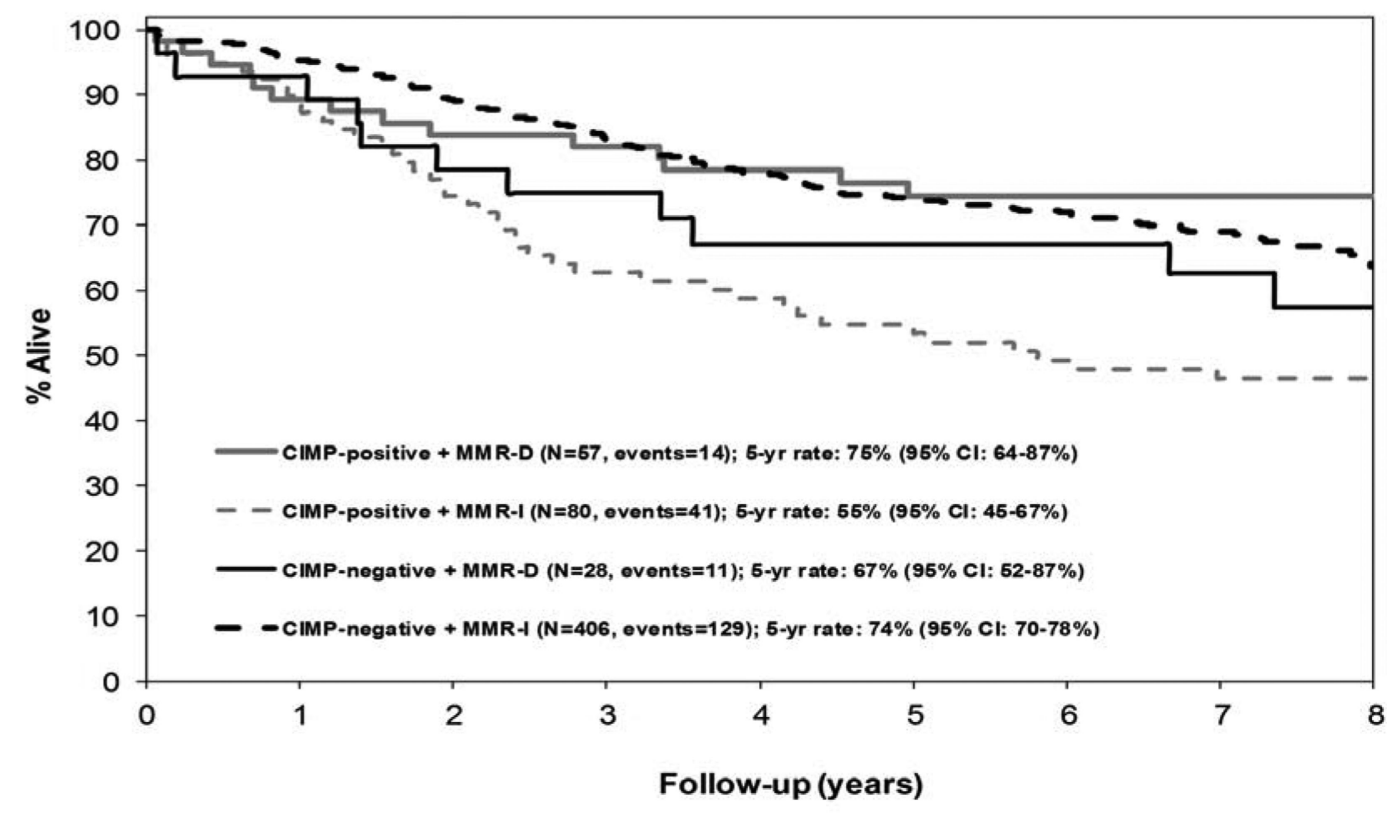

\section{B: Interaction of CIMP status and MMR status for Disease-Free Survival}

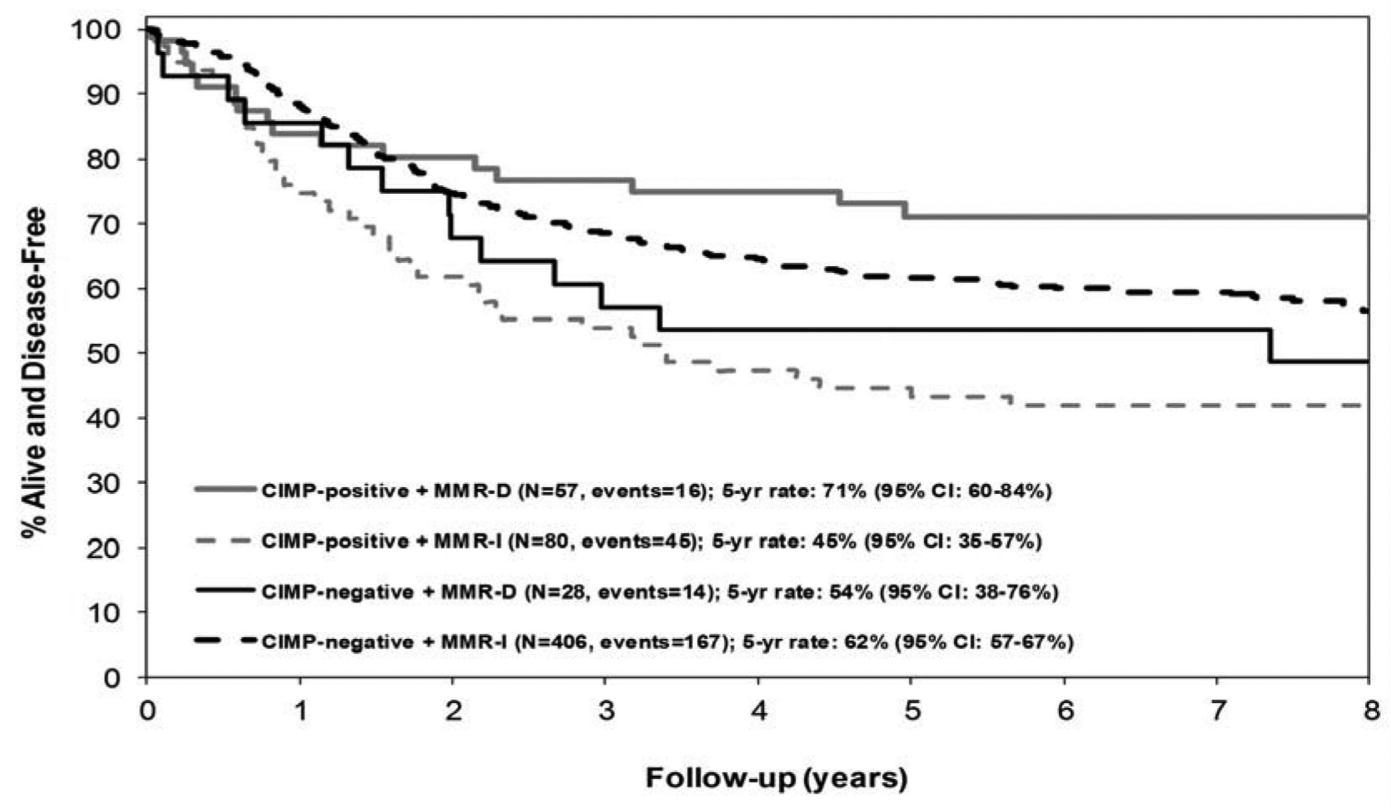

Figure 4. 
In the two-way interaction model between MMR and CIMP, patients with CIMP-positive, MMR-D tumors had improved overall survival compared to CIMP-positive, MMR-I tumors. However, there was no significant overall survival difference by MMR status within patients with CIMP-negative tumors. Disease-free survival results were similar. 


\section{A: Combined CIMP, MMR interaction effect on overall survival for the FU/LV treatment arm}

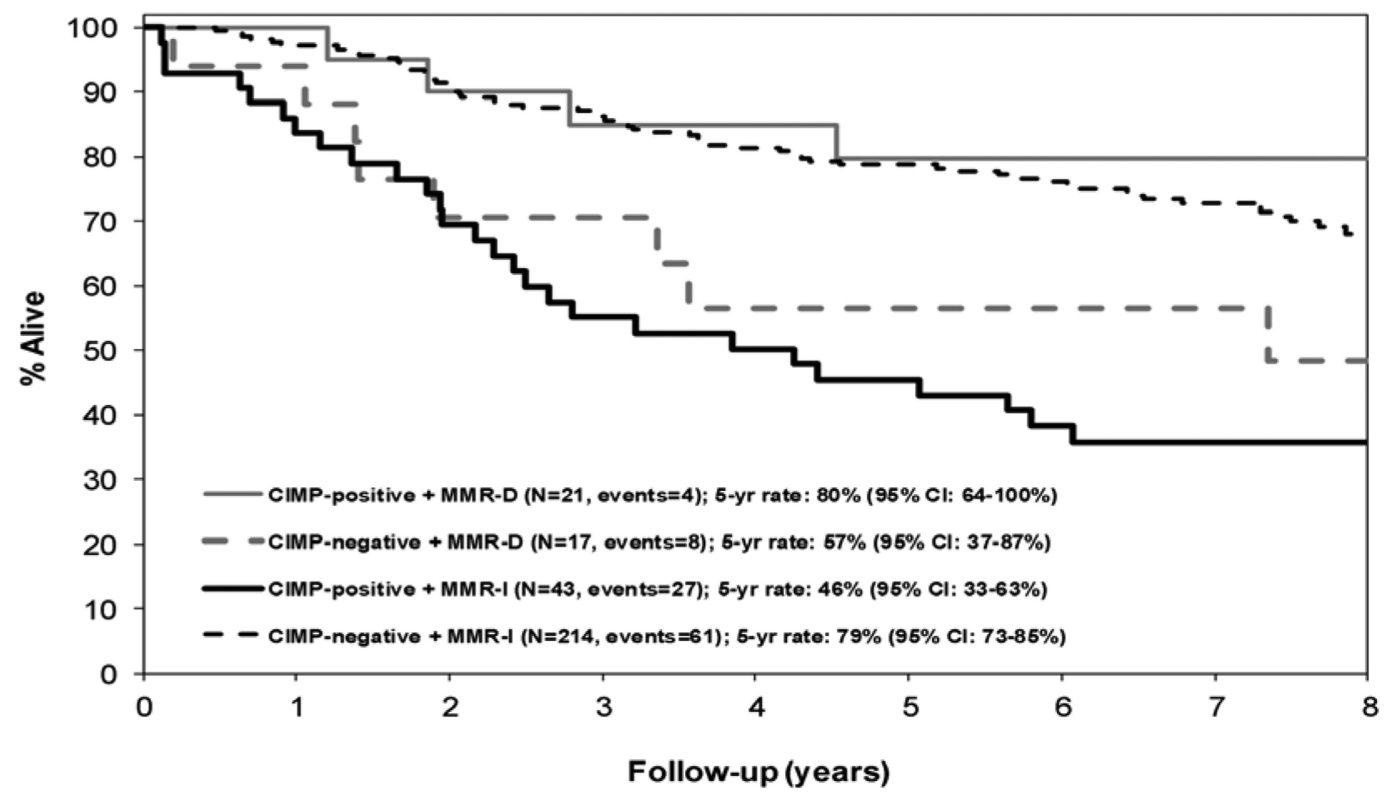

\section{B: Combined CIMP, MMR interaction effect on overall survival for the IFL treatment arm.}

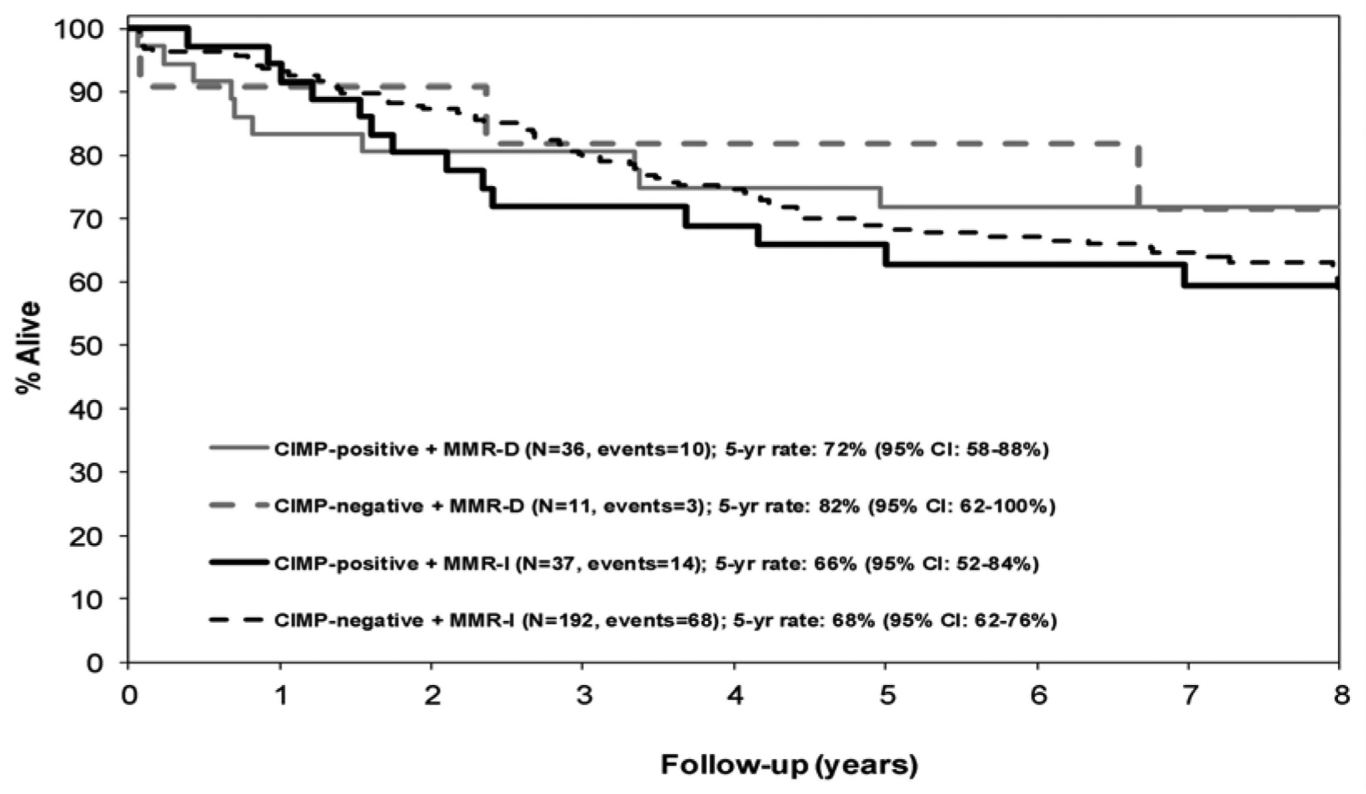




\section{C: Combined treatment, MMR interaction effect on overall survival for the CIMP-negative patients}

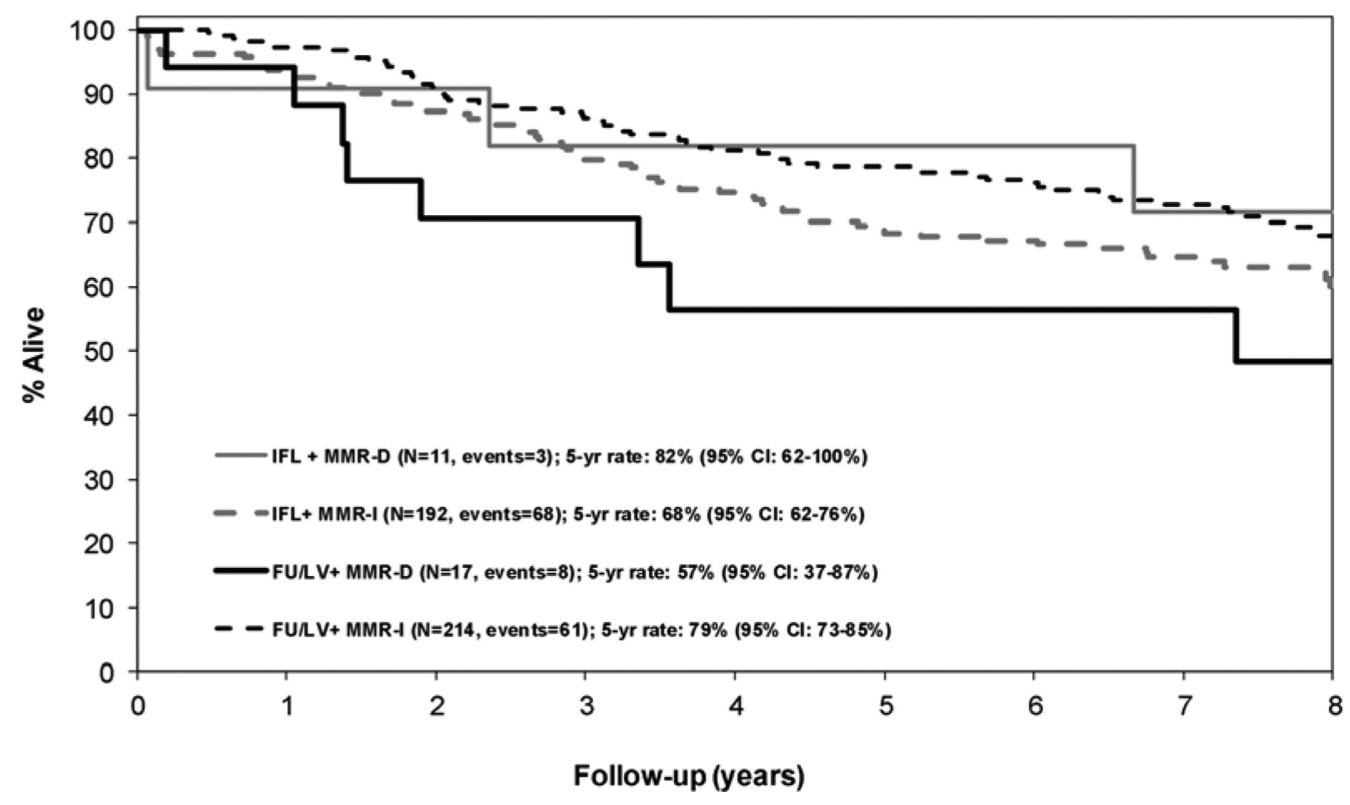

\section{D: Combined treatment, MMR interaction effect on overall survival for the CIMP-positive patients}

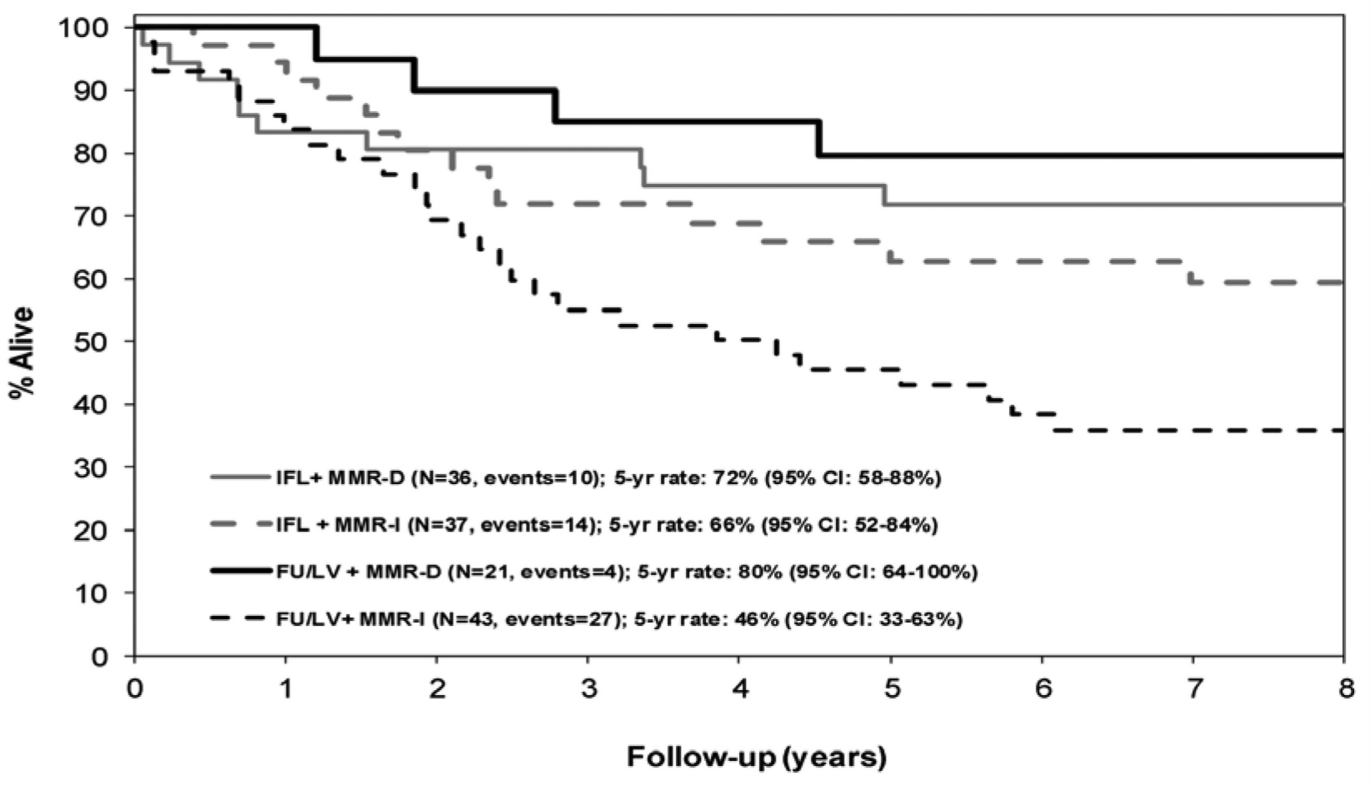

Figure 5.

In the three-way interaction model between CIMP, MMR, and chemotherapy treatment arm, patients with CIMP-negative tumors treated with FU/LV had worse overall survival if they 
were MMR-D (vs. MMR-I). The reverse is true for CIMP-positive patients, where a benefit from FU/LV was observed in MMR-D vs. MMR-I tumors. In the IFL arm, there was no significant difference by CIMP and MMR status. 
Table 1

Demographics of the study population by CIMP status

\begin{tabular}{|c|c|c|c|c|}
\hline & CIMP-Negative $(\mathrm{N}=470)$ & CIMP-Positive (N=145) & Total $(\mathrm{N}=615)$ & p-value \\
\hline Age at study entry & & & & $<0.0001^{b}$ \\
\hline Median & 60 & 68 & 63 & \\
\hline Range & $(24-85)$ & $(29-81)$ & $(24-85)$ & \\
\hline Gender & & & & $0.28^{a}$ \\
\hline Male & $264(56 \%)$ & $74(51 \%)$ & $338(55 \%)$ & \\
\hline Female & $206(44 \%)$ & $71(49 \%)$ & $277(45 \%)$ & \\
\hline Performance status $c$ & & & & $0.03^{a}$ \\
\hline 0 & $355(77 \%)$ & $104(73 \%)$ & $459(76 \%)$ & \\
\hline 1 & $109(24 \%)$ & $37(26 \%)$ & $146(24 \%)$ & \\
\hline 2 & $0(0 \%)$ & $2(1 \%)$ & $2(0.3 \%)$ & \\
\hline Treatment arm & & & & $0.22^{a}$ \\
\hline $\mathrm{FU} / \mathrm{LV}$ & $248(53 \%)$ & $68(47 \%)$ & $316(51 \%)$ & \\
\hline IFL & $222(47 \%)$ & $77(53 \%)$ & $299(49 \%)$ & \\
\hline Tumor Site ${ }^{c}$ & & & & $<0.0001{ }^{a}$ \\
\hline Distal & $233(50 \%)$ & $16(11 \%)$ & $249(41 \%)$ & \\
\hline Proximal & $229(50 \%)$ & $127(89 \%)$ & $356(59 \%)$ & \\
\hline T-Stage $c$ & & & & $0.17^{a}$ \\
\hline $\mathrm{T} 1, \mathrm{~T} 2$ & $59(13 \%)$ & $13(9 \%)$ & $72(12 \%)$ & \\
\hline $\mathrm{T} 3$ & $370(80 \%)$ & $112(79 \%)$ & $482(80 \%)$ & \\
\hline $\mathrm{T} 4$ & $33(7 \%)$ & $16(11 \%)$ & $49(8 \%)$ & \\
\hline Number of nodes sampled $c$ & & & & $0.38^{b}$ \\
\hline Median & 12 & 13 & 12 & \\
\hline Range & $(1-99)$ & $(2-56)$ & $(1-99)$ & \\
\hline Number of Positive Nodes $c$ & & & & $0.13^{b}$ \\
\hline Median & 2 & 3 & 3.0 & \\
\hline Range & $(1-24)$ & $(1-23)$ & $(1-24)$ & \\
\hline Extramural vascular invasion $c$ & & & & $0.44^{a}$ \\
\hline No & $412(9 \%)$ & $124(89 \%)$ & $536(91 \%)$ & \\
\hline Yes & $39(9 \%)$ & $15(11 \%)$ & $54(9 \%)$ & \\
\hline Perineural invasion $c$ & & & & $0.13^{a}$ \\
\hline No & $418(93 \%)$ & $125(89 \%)$ & $543(92 \%)$ & \\
\hline Yes & $33(7 \%)$ & $16(11 \%)$ & $49(8 \%)$ & \\
\hline Lymphovascular invasion $c$ & & & & $<0.0001^{a}$ \\
\hline No & $326(71 \%)$ & $75(53 \%)$ & $401(67 \%)$ & \\
\hline Yes & $131(29 \%)$ & $67(47 \%)$ & $198(33 \%)$ & \\
\hline
\end{tabular}




\begin{tabular}{|c|c|c|c|c|}
\hline & CIMP-Negative ( $\mathrm{N}=470)$ & CIMP-Positive (N=145) & Total $(\mathrm{N}=615)$ & p-value \\
\hline Histologic Grade ${ }^{c}$ & & & & $<0.0001^{a}$ \\
\hline Grade 1,2 & $371(80 \%)$ & $79(55 \%)$ & $450(74 \%)$ & \\
\hline Grade 3,4 & $92(20 \%)$ & $64(45 \%)$ & $156(26 \%)$ & \\
\hline MMR Status ${ }^{c}$ & & & & $<0.0001{ }^{a}$ \\
\hline MMR-I & $406(94 \%)$ & $80(58 \%)$ & $486(85 \%)$ & \\
\hline MMR-D & $28(7 \%)$ & $57(42 \%)$ & $85(15 \%)$ & \\
\hline$B R A F V 600 E^{c}$ & & & & $<0.0001{ }^{a}$ \\
\hline Wild-Type & $425(96 \%)$ & $61(45 \%)$ & $486(84 \%)$ & \\
\hline Mutant & $16(4 \%)$ & $76(56 \%)$ & $92(16 \%)$ & \\
\hline$K R A S^{c}$ & & & & $<0.00011^{a}$ \\
\hline Wild-Type & $261(60 \%)$ & $115(82 \%)$ & $376(65 \%)$ & \\
\hline Mutant & $175(40 \%)$ & $25(18 \%)$ & $200(35 \%)$ & \\
\hline$T P 53^{c}$ & & & & $0.25^{a}$ \\
\hline Wild-Type & $168(55 \%)$ & $57(61 \%)$ & $225(56 \%)$ & \\
\hline Mutant & $140(46 \%)$ & $36(39 \%)$ & $176(44 \%)$ & \\
\hline
\end{tabular}

${ }^{a}$ Chi-Square test p-value

${ }^{b}$ Wilcoxon Rank-Sum test p-value

${ }^{c}$ Missing data on some patients 